\title{
Driving Waveform Design Based on Driving Process Fusion and Black Reference Gray Scale for Electrophoretic Displays
}

\author{
Li Wang *, Pengchang Ma*, Jitao Zhang and Qiming Wan \\ School of Information Engineering, Zhongshan Polytechnic, Zhongshan, China
}

An electrophoretic display (EPD) is a kind of paper display technology, which has the advantages of ultra-low power consumption and readability under strong light. However, in an EPD-driving process, four stages are needed to finish the driving of a pixel erase original images, reset to black state, clear-to-white state, and write a new image. A white reference

OPEN ACCESS

Edited by:

Qiang Xu,

Nanyang Technological University,

Singapore

Reviewed by:

Weimin $L i$

Shenzhen Institutes of Advanced

Technology, CAS, China

Jingling Yan,

Ningbo Institute of Materials

Technology and Engineering, CAS,

China

*Correspondence: Li Wang

wangli_oet@163.com

Pengchang $\mathrm{Ma}$

mpcmyc99@aliyun.com

Specialty section:

This article was submitted to

Optics and Photonics,

a section of the journal

Frontiers in Physics

Received: 10 June 2021

Accepted: 05 July 2021

Published: 09 August 2021

Citation:

Wang L, Ma P, Zhang J and Wan Q (2021) Driving Waveform Design

Based on Driving Process Fusion and

Black Reference Gray Scale for

Electrophoretic Displays.

Front. Phys. 9:723106.

doi: 10.3389/fphy.2021.723106 gray scale can be obtained before writing a new image, and this driving process may take too long for the comfort of reading. In this article, an EPD-driving waveform, which takes the black state as the reference gray, is proposed to reduce the driving time. In addition, the rules of direct current (DC) balance are also followed to prevent the charge from getting trapped in the driving backplane. The driving process is fused and there are two stages in the driving waveform: reset to black state and write the next image. First, the EPD is written to a stable black state according to the original gray scale driving waveform and the black state is used as the reference gray for the next image. Second, the new image is written by the second stage of the new driving waveform. The experimental results show that the proposed driving waveform has a better performance. Compared with the traditional driving waveform which has four stages, the driving time of the new driving waveform is reduced by nearly $50 \%$.

Keywords: electrophoretic display, driving waveform, driving process, reference gray scale, direct current balance

\section{INTRODUCTION}

EPD has become the most widely used paper-like display due to its good market potential. As a reflective display technology, the light reflection characteristic provides it with a paper-like comfortable reading experience [1-4]. In addition, EPDs also have the advantages of ultra-low power consumption and a good bistable characteristic [5, 6]. Therefore, EPDs have been widely used in electronic labels, e-books, etc. But video playback cannot be achieved due to the long response time of EPDs [7]. Particles in microcapsules of EPDs are driven by voltages, and pixels can display different gray scales by adjusting the position of particles [7-10]. The voltage sequence which can drive particles to display gray scales is called the driving waveform. Hence, it is of great significance to reduce the response time and improve the display effect by optimizing the driving waveform design $[11,12]$.

A traditional driving waveform can be divided into four stages: erasing the original image, resetting to the black state, clearing to the white state, and writing a new image [13-15]. When a particle is in a same position for a long time, its mobility is much lower than that of other particles. 
The particle activity can be improved by resetting to the black state and clearing to the white state [16-18]. This driving process may be continued from hundreds of milliseconds to $1 \mathrm{~s}[19,20]$. Therefore, there are some scholars who have conducted a series of research on the driving waveform, and some principles and methods of driving waveform editor are put forward to shorten the driving time or reduce flickers. Kao et al. [21] studied the property of the suspension viscosity, characterized the response latency of the EPD, and put forward a new driving waveform, which set the white gray scale as a reference gray scale, to shorten the driving time and reduce the number of flickers. However, the proposed driving method did not take into account an important factor-DC balance. Moreover, the reference gray scale is unstable. Wang et al. [22] used four kinds of screen update modes to update the EPD according to different images, and it could improve the update speed. However, the form of driving waveforms in four modes was not changed, and it could not improve the refresh speed fundamentally. In a traditional driving waveform, stages of resetting to the black state and clearing to the white state are longer than the other two stages [23,24], so some scholars have studied new driving schemes to reduce the driving time of these two stages. Yi et al. [25] found that high-frequency voltage can be used to activate particles, and the new driving waveform could effectively reduce the ghost image and flicker. But it is difficult to get a stable gray scale display. He et al. [26] proposed a driving waveform based on the optimization of particle activation by analyzing the electrophoresis performance of particles. It can effectively reduce the driving time. However, the DC balance is not considered in the design of the driving waveform, which may cause damage to the EPD [27].

In this article, a new driving waveform, which is based on the black reference gray, is proposed to reduce the driving time. The new driving waveform obeys the rule of DC balance and is divided into two stages: reset to black state and write the new image. The first stage is used to get an accurate reference gray, and some principles are proposed to get a uniform reflectance value for black state at the same time. The second stage is used to write a new gray value by comparing it with the black reference gray.

\section{MICROENCAPSULATED EPD SYSTEM}

The introduction of microcapsule technology is an important breakthrough in EPD technology. There are two kinds of particles in a microcapsule [28]. Here, the microcapsule is a pixel in EPD. It solves the long-term display instability problem in EPD. The

A
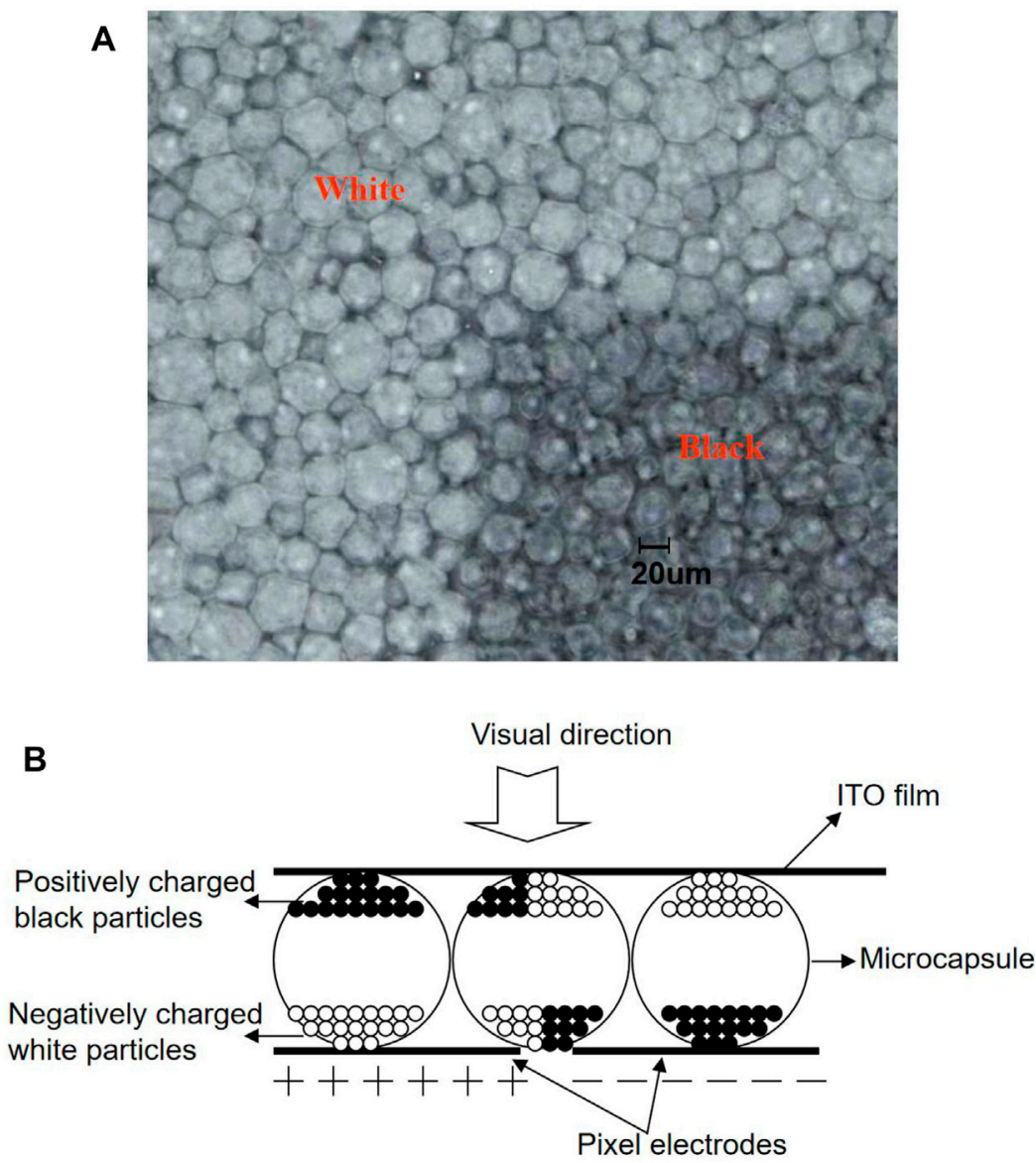

FIGURE 1 | The structure of a microcapsule EPD. (A) An enlargement image of microcapsules in an EPD. (B) EPD model. 
movement and aggregation of particles are limited in microcapsules, and the different gray scales could be realized by controlling the voltage which is applied on electrodes. In the display process, the white $\mathrm{SiO}_{2}$ particles of the EPD are charged with a negative charge and black carbon particles are charged with a positive charge. The structure of an EPD is shown in Figure 1. In Figure 1A, the electrode of a pixel in the upper left part is provided with a negative voltage and the positively charged white particles move upward, the EPD shows white, the lower right part is provided with a positive voltage and the negatively charged black particles move upward, and the EPD shows black.

In the interior of a microcapsule, as is shown in Figure $\mathbf{1 B}$, in order to ensure the good electrophoretic performance of electrophoretic particles, a charged control agent is injected into the display capsule, which can effectively prevent the accumulation of charged particles and deposit on the capsule wall. The display particles in the capsule are mainly affected by gravity, buoyancy, electric power, and the viscosity of nonpolar solvent. In order to avoid the settlement of display particles, gravity is equal to buoyancy, as shown in Eq. 1.

$$
\left[\frac{\left(3 m-4 \pi R^{3} \rho\right) g}{3}\right]=0 .
$$

Here, $m$ is the mass of a particle, $R$ is the radius of a particle, $\rho$ is the density of nonpolar solvents, and $g$ is the gravity constant. The electric force can be expressed by Eq. 2 .

$$
\overrightarrow{F_{E}}=q \vec{E}+\vec{m} \nabla \vec{E}+\frac{1}{6} \nabla(\vec{Q}: \nabla \vec{E})+O\left(\nabla^{3} E\right) .
$$

Here, $\overrightarrow{F_{E}}$ is the electric force, $q$ is the charge of the particle, $\vec{E}$ is electric field, and $\vec{Q}$ is the total charge of particles. The first term of Eq. 2 is the Coulomb interaction between particles and electric field, and the other term is the interaction of dielectric polarization components induced by particles. The viscosity can be expressed by Eq. 3 [29, 30].

$$
\overrightarrow{F_{S}}=6 \pi \eta R \vec{v}
$$

Here, $\overrightarrow{F_{S}}$ is the viscosity, $\eta$ is the viscosity coefficient of the nonpolar solvent, and $\vec{v}$ is the velocity of the particle. $\vec{v}$ can be obtained by solving Eq. 3, and it is shown in Eq. 4 [31].

$$
\vec{v}=\frac{q \overrightarrow{F_{E}}}{6 \pi \eta R}\left(1 \pm e^{-\frac{6 \pi \eta R t}{m}}\right) \text {. }
$$

In general, the charged particle zeta potential could reach a level which could make a particle whose radius is $1 \mu \mathrm{m}$ with $50-100$ charges by using the charge control agent, at the same time, the electrophoretic mobility reaches $10^{-4}-10^{-5} \mathrm{~cm}^{2} / V s$. The expression of response time is shown as Eq. 5 .

$$
T=\frac{6 \pi d^{2} \eta}{V \xi \varepsilon},
$$

where $T$ is the response time, $\mathrm{d}$ is the distance between the common electrode and the pixel electrode, $V$ is the voltage applied between two kinds of electrodes, $\xi$ is the particle zeta potential, and $\varepsilon$ is the dielectric constant of suspension liquid.

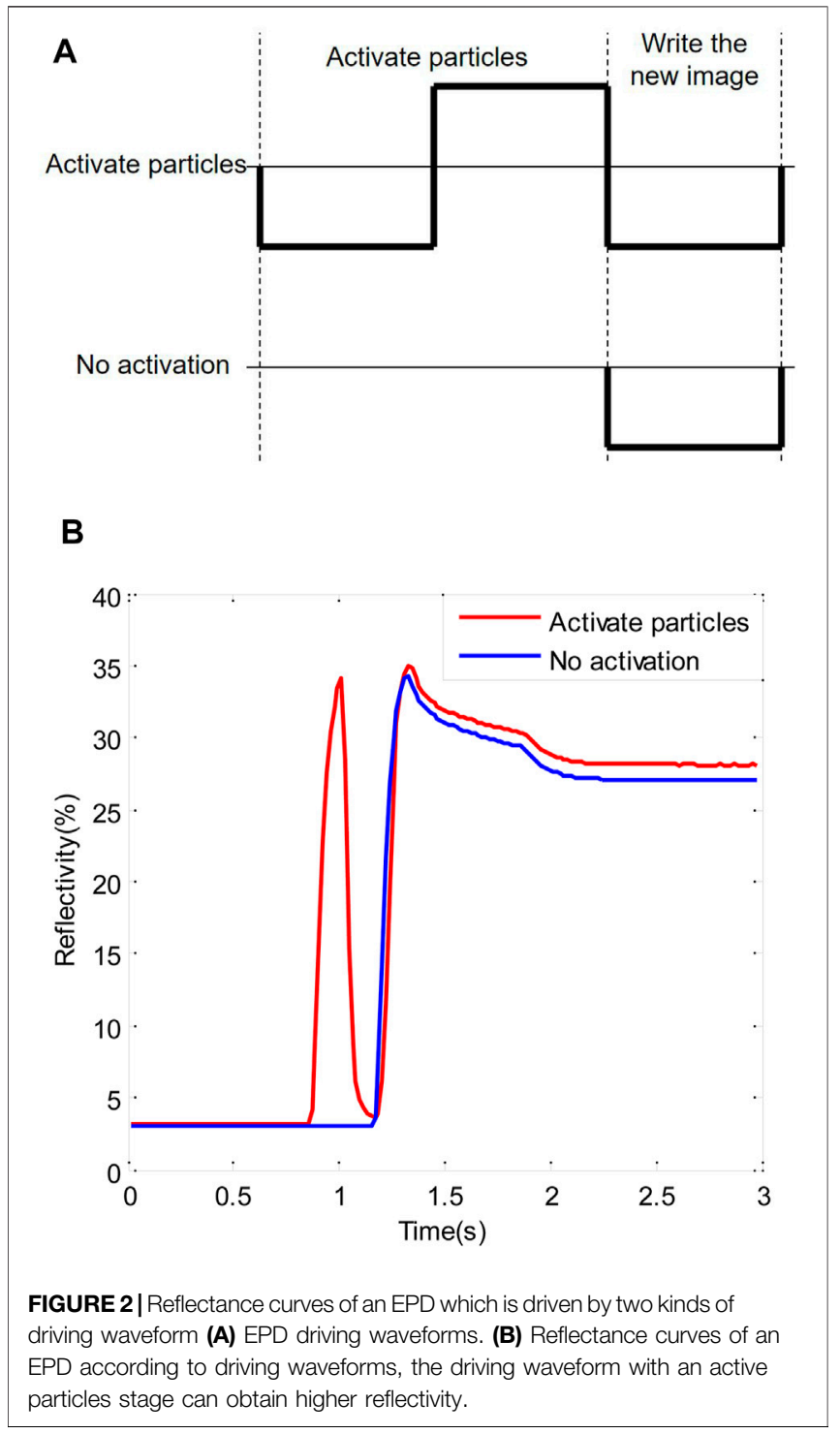

Generally speaking, the voltage of the driving waveform could be set as 15,0 , or $-15 \mathrm{~V}$, and the EPD may spend $1 \mathrm{~s}$ or so on updating the image.

\section{RESULTS AND DISCUSSION}

\section{Particle Performance Analysis}

In the process of driving particles, the EPD may not display a desired gray scale if we drive particles to realize gray scales directly. In the traditional driving waveform, a four-stage driving waveform is used to drive particles to realize gray scales accurately [7]: erase the original image, reset to black state, clear to white state, and write a new image. In a driving waveform, a unit of time is $1 / 50=20 \mathrm{~ms}$ if the frame rate is 50 frames/s, so the time of four stages must be an integer multiple of $20 \mathrm{~ms}$, the second stage and the third stage form a process of activating particles. Then, an accurate white reference gray scale 


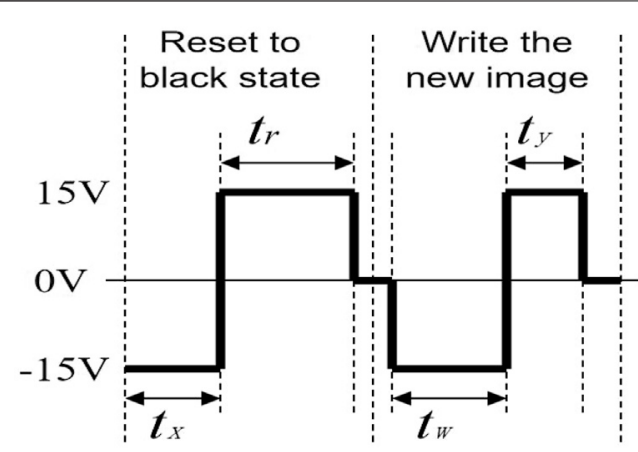

FIGURE 3 | An example of the new driving waveform which includes a resetting to black state stage and a writing the new image stage.

can be obtained at the end point of the third stage. The new image can be displayed in the fourth stage by using the reference gray scale.

In the design process of a driving waveform, the DC balance is a factor which must be considered. In the traditional driving waveform, the time of positive voltage and negative voltage must equal in the one gray scale change path to reach the DC balance.

The process of activating particles is very important in the driving waveform. The particles in microcapsules may be affected by various forces, and the driving force of electric potential must overcome other external forces to drive the particles. However, the resistance increases with the increase of speed. The forces that hinder the movement of particles are particle gravity, viscous resistance of electrophoretic solution, collision between particles, wall collision of microcapsules, Coulomb force between particles, and so on. When the resistance of particles is greater than the electric field force, the particle velocity will decrease. Only after a long enough residence time, the particles can reach a stable state, and the force acting on the particles can reach equilibrium. This reaction time has a direct impact on the refresh speed of the display screen. At this time, the motion ability of the moving particles is obviously lower than that of the activated particles [6]. Therefore, it is necessary to design a longer voltage duration to obtain the same gray level as the driving waveform with the active particle stage. Therefore, at the beginning of the driving waveform, we always use the limited driving time to activate the particles as much as possible. There are two driving waveforms, which are designed to drive the EPDs from a black state to a white state, in Figure 2A. The final reflectivity difference of an EPD which is driven by two driving waveforms are shown in Figure 2B.

\section{Driving Process Fusion in the Driving Waveform}

In this article, a new driving waveform, which is based on the black reference gray, is proposed. In this waveform, the driving process is fused, and there are two stages which include a resetting to black state stage and writing the new image stage. In the first stage, the particles are activated as far as possible, and a stable reflectivity black state is obtained at the end of this stage; then, the black state is taken as the reference gray. In the second stage, the positive voltage and negative voltage can cooperate with each other to realize the display of multilevel gray scales. An example of the new driving waveform is shown in Figure 3.

The second stage of the driving waveform, which could drive the EPD to realize the original image, must be considered in the design of the new driving waveform. For example, $t_{w}$ is the negative voltage driving time and $t_{y}$ is the positive voltage driving time of second stage which could drive the EPD to display the original image. And $t_{x}$ is the negative voltage driving time and $t_{r}$ is the positive voltage driving time of the first stage which could drive the EPD to display a new image. The relationship among four driving times is shown in Eq. 6.

$$
t_{w}-t_{y}=t_{r}-t_{x}
$$

The driving waveform could reach the DC balance if Eq. 6 is obeyed. In the first stage of the driving waveform, there is not enough time for refreshing between the white state and the black state, so the EPD is written to a middle gray scale, and this method could reduce the activation time greatly [8]. The detail design of the driving waveform is shown in Figure 4. For the sake of convenience, B represents black gray, DG represents dark gray, LG represents light gray, and $W$ represents white gray, respectively. In Figure 4, the original gray scale is LG, both $t_{1}$ and $t_{2}$ are square waves whose duty ratio is $50 \%$, so it has no effect on DC balance. $t_{3}$ is used to realize the DC balance and write to black state. At the end point of the first stage, a black state which has a stable reflectance value is obtained. The first stage of driving
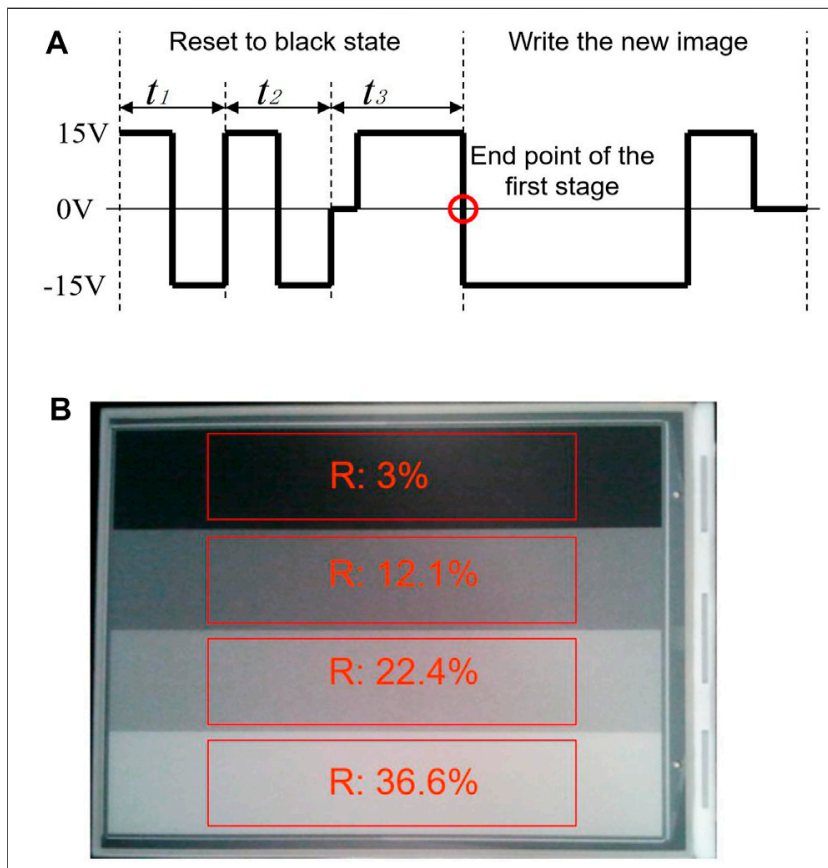

FIGURE 4 | (A) Design details of the new driving waveform, at the end point of the first stage, a black state which has a stable reflectance value can be obtained. (B) The real EPD has four-level gray scales which are driven by the proposed driving waveform. 
waveforms should be filled with a positive voltage when the original gray scale is white, but it cannot get a black gray as the black reference gray. This is because the black reference gray is obtained in the case of activating particles, but there is no space for the activated particles stage when this stage is full of positive voltage. So, the first stage must be prolonged to form a longer time of positive voltage to get the black reference gray. The prolonged part could occupy the driving time of the second stage, or else, the driving waveform must be prolonged a little to meet the requirement.

\section{Display Effect of the Proposed Driving Waveform}

We carried out a driving experiment on an EPD device according to the proposed driving waveform, and the result is shown in Figure 4B. The experimental results showed that the driving waveform could realize multilevel gray scales. Among them, four testing areas are marked, the reflectivity of the first testing area is $3 \%$, the second testing area is $12.1 \%$, the third testing area is $22.4 \%$, and the fourth testing area is $36.3 \%$. According to the principle of gamma correction, the human eye sensitive to brightness transformation when the brightness is low. So, the change of the reflectivity value is nonlinear.

\section{CONCLUSION}

In this article, a new driving waveform based on two stages is proposed, and the driving process of gray scales is effectively fused. The first stage of the driving waveform can erase the original image, and the second stage is used to realize a multilevel gray scales display. In addition, the black state is used as the reference gray scale according to the mechanical analysis of

\section{REFERENCES}

1. Heikenfeld J, Drzaic P, Yeo J-S, and Koch T. Review Paper: A Critical Review of the Present and Future Prospects for Electronic Paper. J Soc Inf Display (2011) 19(2):129-56. doi:10.1889/JSID19.2.129

2. Meng X, Wen T, Qiang L, Ren J, and Tang F. Luminescent Electrophoretic Particles via Miniemulsion Polymerization for Night-Vision Electrophoretic Displays. ACS Appl Mater Inter (2013) 5(9):3638-42. doi:10.1021/am400103d

3. Lee J-K, Kim S-S, Park Y-I, Kim C-D, and Hwang Y-K. In-cell Adaptive Touch Technology for a Flexible E-Paper Display. Solid-state Electron (2011) 56(1): 159-62. doi:10.1016/j.sse.2010.10.008

4. Yi Z, Huang Z, Lai S, He W, Wang L, and Chi F. Driving Waveform Design of Electrowetting Displays Based on an Exponential Function for a Stable Grayscale and a Short Driving Time. Micromachines (2020) 11(3):313. doi:10.3390/mil1030313

5. Kao W-C, Wu G-F, Shih Y-L, Chang W-T, Lin F-S, and Hsieh Y-J. Design of Real-Time Image Processing Engine for Electrophoretic Displays. J Display Technol (2011) 7(10):556-61. doi:10.1109/JDT.2011.2159360

6. Li W, Wang L, Zhang T, Lai S, Liu L, and He W. Driving Waveform Design with Rising Gradient and Sawtooth Wave of Electrowetting Displays for Ultralow Power Consumption. Micromachines (2020) 11(2):145. doi:10.3390/ mil1020145 particles in microcapsules. The driving waveform proposed by us has the advantages of short driving time and less voltage conversion times. It can effectively reduce the flicker sense and improve the reading comfort while shortening the screen switching time. What is more, the DC balance rule is applied to the design, which can effectively prevent the display breakdown caused by charge trapping. So, the proposed driving waveform provides a reference for the continuous improvement of the EPD display quality.

\section{DATA AVAILABILITY STATEMENT}

The original contributions presented in the study are included in the article/Supplementary Material; further inquiries can be directed to the corresponding authors.

\section{AUTHOR CONTRIBUTIONS}

LW designed this project. PM and JZ carried out most of the experiments and data analysis. QW performed part of the experiments and helped with discussions during manuscript preparation. All authors have read and agreed to the published version of the manuscript.

\section{FUNDING}

This research was funded by the Key Projects of the Second Batch of Social Welfare and Basic Research in Zhongshan City (No. 2020B2021), the High-level Scientific Research Startup Project in Zhongshan Polytechnic (No. KYG2104), and the Special Projects in Key Fields of Colleges and Universities in Guangdong Province in 2020 (No. 2020ZDZX3083).

7. Bai PF, Hayes RA, Jin M, Shui L, Yi ZC, and Wang L. Review of Paper-Like Display Technologies (Invited Review). Prog Electromagn Res C (2014) 147: 95-116. doi:10.2528/PIER13120405

8. Zeng W, Yi Z, Zhao Y, Zeng W, Ma S, and Zhou X. Design of Driving Waveform Based on Overdriving Voltage for Shortening Response Time in Electrowetting Displays. Front Phys (2021) 9:642682. doi:10.3389/ fphy.2021.642682

9. Kao W-C, Liu C-H, Liou S-C, Tsai J-C, and Hou G-H. Towards Video Display on Electronic Papers. J Display Technol (2016) 12(2):129-35. doi:10.1109/ JDT.2015.2469539

10. Yi Z, Feng H, Zhou X, and Shui L. Design of an Open Electrowetting on Dielectric Device Based on Printed Circuit Board by Using a Parafilm M. Front Phys (2020) 8:193. doi:10.3389/fphy.2020.00193

11. Kao W-C. Electrophoretic Display Controller Integrated with Real-Time Halftoning and Partial Region Update. J Display Technol (2010) 6(1):36-44. doi:10.1109/JDT.2009.2030774

12. Yi Z, Zeng W, Ma C, Feng H, Yang J, and Liu L. A Real-time Touch Control System Design Based on Field-programmable Gate Array via Optimizing Bresenham Algorithm for Electrowetting Displays. J Soc Inf Display (2021) 29: 573-83. doi:10.1002/jsid.1001

13. Kao W-C, Chen H-Y, Liu Y-H, and Liou S-C. Hardware Engine for Supporting Gray-Tone Paintbrush Function on Electrophoretic Papers. J Display Technol (2014) 10(2):138-45. doi:10.1109/JDT.2013.2289364 
14. Lu C-M, and Wey C-L. A Controller Design for Micro-Capsule Active Matrix Electrophoretic Displays. J Display Technol (2011) 7(8):434-42. doi:10.1109/ JDT.2011.2142173

15. Yi Z, Zeng W, Ma S, Feng H, Zeng W, and Shen S. Design of Driving Waveform Based on a Damping Oscillation for Optimizing Red Saturation in Three-Color Electrophoretic Displays. Micromachines (2021) 12(2):162. doi:10.3390/mi12020162

16. Yang S-H, Lin F-C, Huang Y-P, Shieh H-PD, Yang B-R, and Chang M-J. P-83: Ghosting Reduction Driving Method in Electrophoretic Displays. SID Int Symp Dig Tech Pap (2012) 43(1):1361-4. doi:10.1002/j.2168-0159.2012.tb06057.x

17. Yi Z, Liu L, Wang L, Li W, Shui L, and Zhou G. A Driving System for Fast and Precise Gray-Scale Response Based on Amplitude-Frequency Mixed Modulation in TFT Electrowetting Displays. Micromachines (2019) 10(11): 732. doi:10.3390/mi10110732

18. Li WC, and Keh HJ. Electrophoretic Mobility of Charged Porous Shells or Microcapsules and Electric Conductivity of Their Dilute Suspensions. Colloids Surf A: Physicochemical Eng Aspects (2016) 497:154-66. doi:10.1016/ j.colsurfa.2016.02.028

19. Wang L, Yi Z, Jin M, Shui L, and Zhou G. Improvement of Video Playback Performance of Electrophoretic Displays by Optimized Waveforms with Shortened Refresh Time. Displays (2017) 49:95-100. doi:10.1016/ j.displa.2017.07.007

20. Yi Z, Feng W, Wang L, Liu L, Lin Y, and He W. Aperture Ratio Improvement by Optimizing the Voltage Slope and Reverse Pulse in the Driving Waveform for Electrowetting Displays. Micromachines (2019) 10(12):862. doi:10.3390/ mi10120862

21. Kao W-C, Chang W-T, and Ye J-A. Driving Waveform Design Based on Response Latency Analysis of Electrophoretic Displays. J Display Technol (2012) 8(10):596-601. doi:10.1109/JDT.2012.2205896

22. Wang Z, and Liu Z. The Key Technology of Ereader Based on Electrophoretic Display. In: 2010 2nd International Conference on Software Technology and Engineering, San Juan, PR, October 3-5, 2010 (2010). 333-6. doi:10.1109/ ICSTE.2010.5608873

23. Shen S, Gong Y, Jin M, Yan Z, Xu C, and Yi Z. Improving Electrophoretic Particle Motion Control in Electrophoretic Displays by Eliminating the Fringing Effect via Driving Waveform Design. Micromachines (2018) 9(4): 143. doi:10.3390/mi9040143

24. Zeng W, Yi Z, Zhou X, Zhao Y, Feng H, and Yang J. Design of Driving Waveform for Shortening Red Particles Response Time in Three-Color Electrophoretic Displays. Micromachines (2021) 12(5):578. doi:10.3390/ mi12050578
25. Yi Z-C., Bai P-F., Wang L, Zhang X, and Zhou G-F. An Electrophoretic Display Driving Waveform Based on Improvement of Activation Pattern. J Cent South Univ (2014) 21(8):3133-7. doi:10.1007/s11771-014-2285-9

26. He W, Yi Z, Shen S, Huang Z, Liu L, and Zhang T. Driving Waveform Design of Electrophoretic Display Based on Optimized Particle Activation for a Rapid Response Speed. Micromachines (2020) 11(5):498. doi:10.3390/mil1050498

27. Duan F, Bai P, Henzen A, and Zhou G. Driving Waveform Optimization in electrophoretic Display Based on DC-balance. Chin J Liquid Crystals Displays (2016) 31(10):943-8. doi:10.3788/YJYXS20163110.0943

28. Bert T, and De Smet H. Dielectrophoresis in Electronic Paper. Displays (2003) 24(4-5):223-30. doi:10.1016/j.displa.2004.01.009

29. Kang H-L, Kim CA, Lee S-I, Shin Y-K, Lee Y-H, and Kim Y-C. Analysis of Particle Movement by Dielectrophoretic Force for Reflective Electronic Display. J Display Technol (2016) 12(7):747-52. doi:10.1109/ JDT.2016.2524023

30. Crowder TM, Rosati JA, Schroeter JD, Hickey AJ, and Martonen TB. Fundamental Effects of Particle Morphology on LungDelivery: Predictions of Stokes'Law and the Particular Relevance to Dry Powder Inhaler Formulation and Development. Pharm Res (2002) 19(3):239-45. doi:10.1023/A:1014426530935

31. He W, Yi Z, Shen S, Huang Z, Liu L, and Zhang T. Driving Waveform Design of Electrophoretic Display Based on Optimized Particle Activation for a Rapid Response Speed. Micromachines (2020) 11(5):498. doi:10.3390/mi11050498

Conflict of Interest: The authors declare that the research was conducted in the absence of any commercial or financial relationships that could be construed as a potential conflict of interest.

Publisher's Note: All claims expressed in this article are solely those of the authors and do not necessarily represent those of their affiliated organizations, or those of the publisher, the editors and the reviewers. Any product that may be evaluated in this article, or claim that may be made by its manufacturer, is not guaranteed or endorsed by the publisher.

Copyright (c) 2021 Wang, Ma, Zhang and Wan. This is an open-access article distributed under the terms of the Creative Commons Attribution License (CC BY). The use, distribution or reproduction in other forums is permitted, provided the original author(s) and the copyright owner(s) are credited and that the original publication in this journal is cited, in accordance with accepted academic practice. No use, distribution or reproduction is permitted which does not comply with these terms. 